\title{
A Comparative Study on Different Types of Spectrum Sensing Techniques in Centralized Cognitive Cellular Network
}

\author{
Arpita Mandal \\ Heritage Institute of Technology \\ R.C-1/2, Raghunathpur Kolkata-700059
}

\author{
Sabyasachi Chatterjee \\ Heritage Institute of Technology \\ Chowbaga Road, Anandapur PO: East Kolkata, \\ Township, Kolkata- 700-107
}

\begin{abstract}
The shortage of spectrum is increasing day by day. To minimize this problem, dynamic spectrum access (DSA) technique is adopted by Cognitive Radio Technology. Spectrum sensing is an important technique of secondary users or cognitive users to scan the spectrum holes or white spaces. In this paper, they have discussed the sensing method in a Centralized Cognitive Cellular Network. They have also performed a hypothesis test for different values of Probability of Successful Detection and Probability of false alarm while operating in the low Signal-to-Noise Ratio. This paper basically explains different types of spectrum sensing methods graphically. From the graphical analysis, it has been observed that matched filter is the best spectrum sensing method with minimum local sensing time and less spectrum sensing error..
\end{abstract}

\section{Keywords}

Dynamic Spectrum Access; Spectrum Sensing; Centralized Cognitive Cellular Network; Spectrum Sensing Technique; Matched Filter; Local Sensing Time; Spectrum Sensing Error.

\section{INTRODUCTION}

Radio spectrum is a valuable commodity and it is natural resource of a communication system. Due to the increase in large number of subscriber and wireless services this radio spectrum is getting crowded day by day. The International Telecommunications Union (ITU) on the year 2020 has predicted that for mobile communication system the demand for spectrum is on the order of $1280-1720 \mathrm{MHz}$ [1]. In contrast, the total amount of spectrum currently occupied by mobile communication is around $230-430 \mathrm{MHz}$, depending on the geographic region [2]. As they know that cellular network is limited by the static allocated of spectrum. However, the real situation with these allocated bands is that most of time these are underutilized with large spectrum holes at different geographic location. To overcome this problem with the means of improved utilization of spectrum, the Defence Advanced Research Projects Agency (DARPA) introduces the dynamic spectrum access (DSA) [3] networking that shifts the communication paradigm from the traditional fixed spectrum access to the Dynamic spectrum access in Cognitive Radio (CR) technology. The cognitive radio concept dates back to 1998 when the idea was first conceived by Sir Joseph Mitola III at the Royal Institute of Technology in Stockholm. Later in 1999 it was presented officially in an article by Joseph Mitola and Gerald Q. Maguire, Jr. To deal with this problem of underutilized spectrum and spectrum allocation congestion cognitive radio approach [4] senses its local spectrum environment, identify the spectrum holes in radio spectrum space to make autonomous decision of spectrum access. Here, they have used the concept of dynamic spectrum access to improve the spectrum utilization efficiency resulting in easier and flexible spectrum access for future wireless network. Cognitive Radio is an enabling technology in which the secondary users are allowed to use the licensed bands without causing interference to the licensed or primary users [5][6][7].There are many steps in the process of spectrum access, such as Spectrum Sensing, spectrum management, spectrum mi-gration and spectrum sharing [8].Cognitive Radio consists of four functional block: Spectrum Sensing. It will sense the presence of spectrum holes in a radio environment. Spectrum Management. It will predict the time for the availability of spectrum holes for the use of cognitive radio user in the absence of primary user. Spectrum Sharing. It will distribute the spectrum holes among the Cognitive Radio user. Spectrum Mi-gration. It will help for the mobility to some better available spectrum.

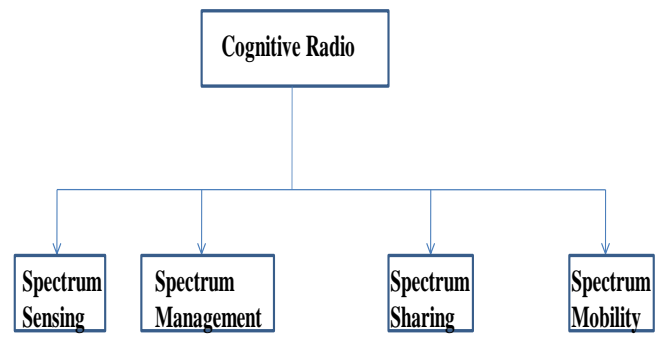

Figure 1: Functional block of Cognitive Radio

The primary user will occupy the spectrum band. By the process of spectrum sensing it will sense its environment and if there is any unoccupied spectrum band or vacant spaces which they have called as spectrum holes then secondary user will occupy those spectrum band as long as the primary user will not become active on that band. Spectrum Sensing can be classified into Centralized or Infrastructure-based Cognitive Cellular network and Distributed or Infrastructure-less Cognitive Cellular network.

In this paper, they have discussed best possible spectrum sensing technique for centralized cognitive cellular network. The remainder of the paper is organized as follows: Section II explains the channel allocation procedure with computational timing calculation of centralized cellular cognitive radio network. Section III defines mathematical testing model of spectrum sensing scheme. Simulation and graphical analysis is performed in section IV. Section V concludes the paper. 


\section{CENTRALIZED COGNITIVE CELLULAR NETWORK}

In centralized or infrastructure-based network there is a fusion centre or base station which will control and coordinate the activity of cognitive radio user. It will also gather the spectrum sensing result by the Cognitive Radio user. The fusion centre will sense its environment and check whether the Received Signal Strength Indicator (RSSI) value is greater than $18 \mathrm{db}$ or not. One CR user will communicate with other $\mathrm{CR}$ user via the fusion centre. Hence it will reduce the delay time and the back off window time.

\begin{tabular}{|l|l|l|l|l|l|l|l|l|l|l|l|l|}
\hline BW1 & BW2 & BW3 & BW4 & BW5 & BW7 \\
\hline
\end{tabular}

\section{Figure 2: Array of Bandwidth}

There is an array of bandwidth. Using hill climbing approach one bandwidth is selected and checked whether the RSSI value is greater than $18 \mathrm{db}$ or not.

RSSI > 18db [No sensing and shift to other bandwidth] RSSI $<18 \mathrm{db}$ [Sensing takes place]

If sensing takes place in a certain bandwidth then after waiting for Differential Inter Frame Space (DIFS), CR1 user will send Request to Carrier Frequency (RCF) command to the fusion centre. It includes the duration in terms of time limit required for the whole data transmission and Cognitive Network Address (CNA). Then the fusion centre will sense its radio environment for reliable carrier frequency. The information is broadcasted along with CR1 Cognitive Network Address (CNA), CR2 Cognitive Network Address (CNA) using Request to Frequency switch (RFS) command. But the CR1 and CR2 will respond because only its CNA is available in the broadcasted information. Then after Short Inter Frame Space (SIFS) CR2 will replies with ACK to CR1 and CR1 and CR2 will start communicating.

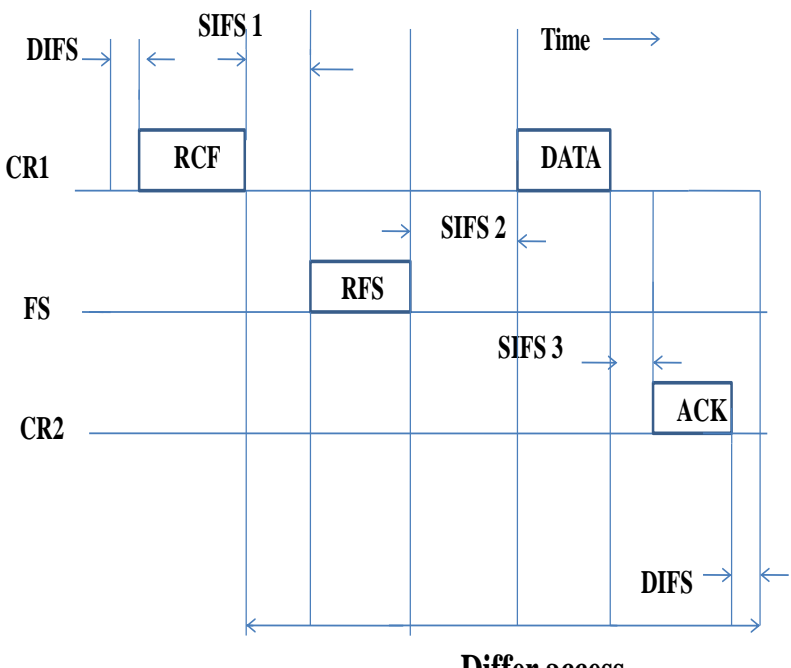

Figure 3: Spectrum Sensing process

Let us consider this total sensing time as T. CR1 and CR2 is situated at a distance of $\mathrm{R}$ meter radius. $\mathrm{CR} 1$ and $\mathrm{CR} 2$ will send their sensing result with their CNA to the fusion centre.

$$
\mathrm{T}=\mathrm{TC}+\mathrm{TS}
$$

Here, TC is the cooperation overhead and TS is the local sensing time.

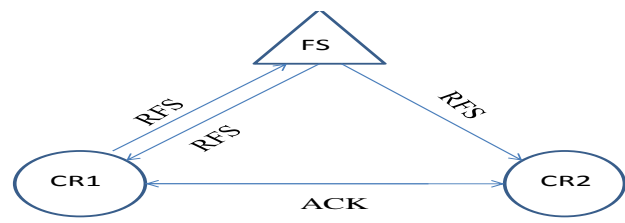

Figure 4: Centralized Cognitive Radio environment

The local sensing time TS is given by:

$$
\mathrm{TS}=\text { ts } \mathrm{N}
$$

Where ts is the sampling interval and $\mathrm{N}$ is the sample size.

$$
\mathrm{T}_{\mathrm{C}}=\frac{\mathrm{I} R}{\sqrt{2 C}}
$$

Where I is the cognitive user and $\mathrm{C}$ is the speed of light.

They have adopted different spectrum sensing technique to sense the radio environment and detect the presence of PU. Using these equations, they have mathematically calculated the value of $\mathrm{T}$ and found that it is minimum in case of MD. They have also shown the variation in the value of TS for different spectrum sensing technique with graphical analysis.

\section{SPECTRUM SENSING TECHNIQUE}

In this paper, they are mainly working with cooperative spectrum sensing method. Cognitive user can occupy a vacant space only if there is no existence of primary user. When primary user is not present to use a particular spectrum, that place is known as spectrum hole. Spectrum hole is a frequency band that is assigned to the PUs but is not utilized at a certain time and location [9], [10], [11]. In order to avoid interference between primary user and spectrum hole, cognitive user sense its environment and allocate the vacant bands for the further use within a certain time limit. This process is called Spectrum Sensing. Spectrum sensing is the task of obtaining awareness about the spectrum usage and existence of primary users in a geographical area. This awareness can be obtained by using geolocation and database, by using beacons, or by local spectrum sensing at cognitive radios [12]-[14]. This method of sensing is done by different types of spectrum sensing technique: Energy-based Spectrum Sensing, Wavelet Transform Sensing, Cyclostationary-based Sensing and Matched filter Spectrum Sensing method. Selection of sensing parameters brings about a tradeoff between the speed (sensing time) and reliability of sensing. Sensing frequency is also an important parameter for spectrum allocation which is affected due to interference between primary user and spectrum hole. Sensing time for detection is always needed to be reduced to maximize the availability of throughput for cognitive user and improves channel efficiency. Sensing time can be reduced using hillclimbing approach. In this method, they will sense only those spectrum of certain bandwidth where there is no interference and use it as the next proposed solution.

\subsection{Energy-based Spectrum Sensing}

It is the simplest method for resource allocation and detection of primary users in the radio environment [4]. Energy detector based approach, also known as radiometry or periodogram, is the most common way of spectrum sensing because of its low computationalandimplementationcomplexities[15],[16],[17],[ 
18].The ED is computationally efficient and could also be used conventionally with analog and digital signals. It is a non coherent detection method.

\subsection{Wavelet-based Spectrum Sensing}

The spectrum is decomposed into smaller sub-bands to detect edges in power spectral density.

\subsection{Cyclostationary-based Spectrum Sensing} Cognitive Radio can distinguish noise and user signal by analyzing its periodicity [4]. The periodicity is embedded in sinusoidal carrier.

\subsection{Matched filter Spectrum Sensing}

This method [3] is the optimal linear filter for maximizing signal-to-noise ratio (SNR) in the presence of additive white Gaussian noise. This is commonly used in Radar technology.

\section{HYPOTHESIS TEST FOR SPECTRUM SENSING SCHEME}

They have taken two hypothesis test. Let us assume that the received signal has the following form

$$
y[n]=s[n]+\omega[n]
$$

For the two hypothesis $\mathcal{H} 0$ i.e null hypothesis and $\mathcal{H} 1$ i.e alternate hypothesis.

$$
\begin{aligned}
& \mathcal{H} 0: y[n]=\omega[n] \quad \text { (No transmission of signal) } \\
& \mathcal{H} 1: y[n]=s[n]+\omega[n] \quad \text { (Signal is transmitted) }
\end{aligned}
$$

Where $s[n]$ is the signal to be detected with zero mean and unit variance, i.e $\sigma \mathrm{u} 2=1, \omega[\mathrm{n}]$ is Additive white Gaussian noise (AWGN) with zero mean and variance as $\sigma \mathrm{w} 2$.

Under hypothesis $\mathcal{H} 0$, it is considered as probability of false alarm that is the considered frequency is occupied when it is actually not.

$$
\mathrm{P}_{\mathrm{F}}(\lambda)=Q\left\{\left(\frac{\lambda}{\sigma_{u}^{2}}-1\right) \sqrt{N}\right.
$$

Under hypothesis $\mathcal{H} 1$, it is considered as probability of detection that is the considered frequency signal is truly present.

$$
\mathrm{P}_{\mathrm{D}}(\lambda)=Q\left\{\left(\frac{\lambda}{\sigma_{u}^{2}}-\gamma-1\right) \sqrt{\frac{N}{2 \gamma+1}}\right.
$$

Using this two equation, they will plot a cumulative distribution function of Probability of detection versus probability of false alarm for the three types of spectrum sensing technique. Here $\lambda$ is the decision threshold which is constant for all spectrum sensing method and is selected to find an optimum balance between PD and PF. N is the sample size, $\sigma \mathrm{u} 2$ is the variance for signal power, $\gamma$ is the received signal to noise ratio (SNR). The Miss-detection probability is given as

$$
\operatorname{Pm}(\lambda)=1-\operatorname{Pd}(\lambda)
$$

They will draw a graph of probability of sensing versus SNR, probability of detection versus SNR for the three spectrum sensing method. The PD value always decreases as the Pf value increases.

\section{SIMULATION AND RESULTS}

They have done a comparative study on different types of Spectrum Sensing method such as Energy detection, Cyclostationary detection and matched filter detection.

To get the simulation result, they have considered $40 \mathrm{~m}$ as the value of distance $(R)$ between the cognitive radio user, $3 * 108 \mathrm{~m} / \mathrm{s}$ as the speed of light, 0.9 as target probability for (PD) , 0.1 as target probability for false alarm (PF), $\lambda$ as decision threshold, 1 as variance $(\sigma \mathrm{u} 2)$.The sensing phenomenon is mainly done by fusion centre by applying different sensing technique. They have calculated the value of Probability of detection, Local sensing time by varying the sample size $(\mathrm{N})$ for different types of sensing method

Using all the above parameter, they have shown our simulation result by doing a comparative study on different types of spectrum sensing method and tried to conclude the best sensing method.

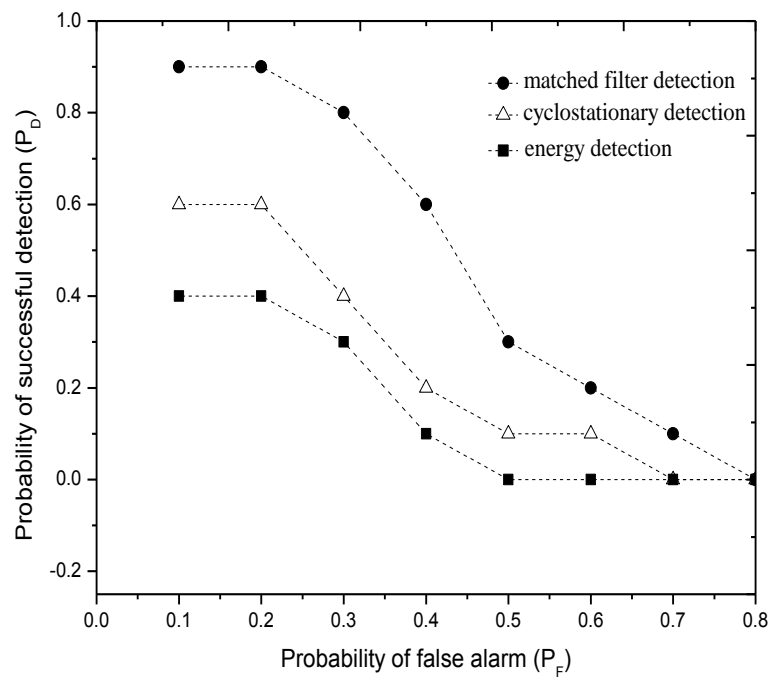

Figure1: Probability of successful detection versus Probability of false alarm

This figure represents the Probability of Successful Detection (PD) versus Probability of false alarm (PF) for different Spectrum Sensing method. It shows how the value of PD decreases for different values of PF.

In energy detection spectrum sensing method, the signal is detected by comparing the output of energy detector with threshold $\lambda$ which is constant. They are working here with low SNR value and in the figure they can see it shows a poor performance of spectrum. This is the reason in ED it is hard to distinguish between the PU signal from the CR signal. In ED the threshold value depends on noise variance which can be taken as $\sigma w 2$. So any estimation error in noise power can cause a significant performance loss. And graphically they can also see that its PD value is much less than the other two sensing method.

In Cyclostationary sensing method it has the property of periodicity of signal. The periodicity is embedded in sinusoidal carrier. This property of periodicity in cyclostationary signal provides the statistics and autocorrelation or spectral correlation function. This autocorrelation function can easily show a likely hood of signal as the signal is periodic. It requires a prior knowledge of PU signal but it can able to distinguish the PU signal from $\mathrm{CR}$ signal and also from noise which has no correlation. 
Graphically they can also see it shows a better performance of PD in low SNR than energy detection method.

In matched filter spectrum sensing method, it works well in low SNR and shows the best performance than the other two sensing method. MFD also requires less sensing time for detection as the sample value i.e $\mathrm{N}$ grows as o(1/SNR) to meet a certain target probability of detection that is the maximum value of PD and PF. Hence in this method they have got maximum throughput and channel efficiency. The $\mathrm{PD}$ is much more and therefore it is the best spectrum sensing method.

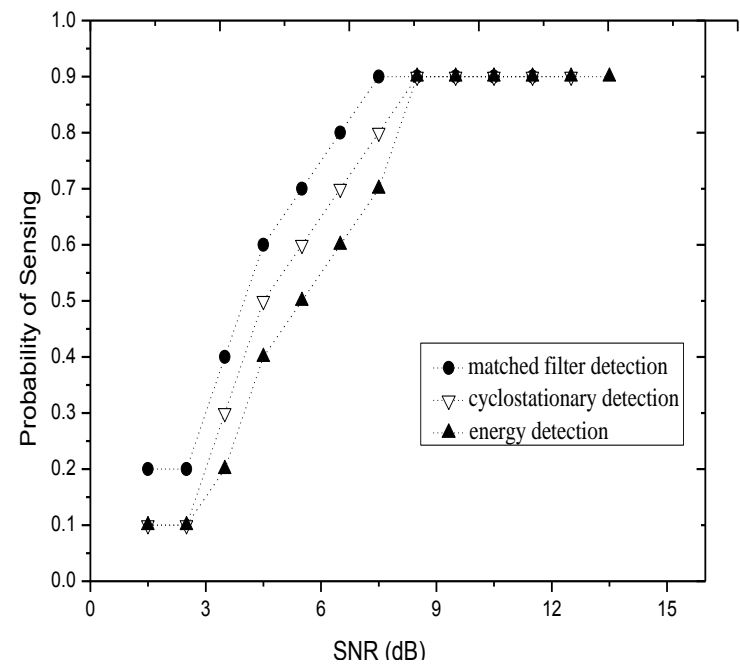

Figure 2: Probability of sensing versus SNR

This figure represents the probability of sensing versus SNR for different Spectrum Sensing methods. As they can see, the probability of sensing increases for different SNR values. The probability of sensing for matched filter is much more than the other two sensing method. Hence, using matched filter sensing technique they can sense the environment properly and shows a good performance of spectrum.

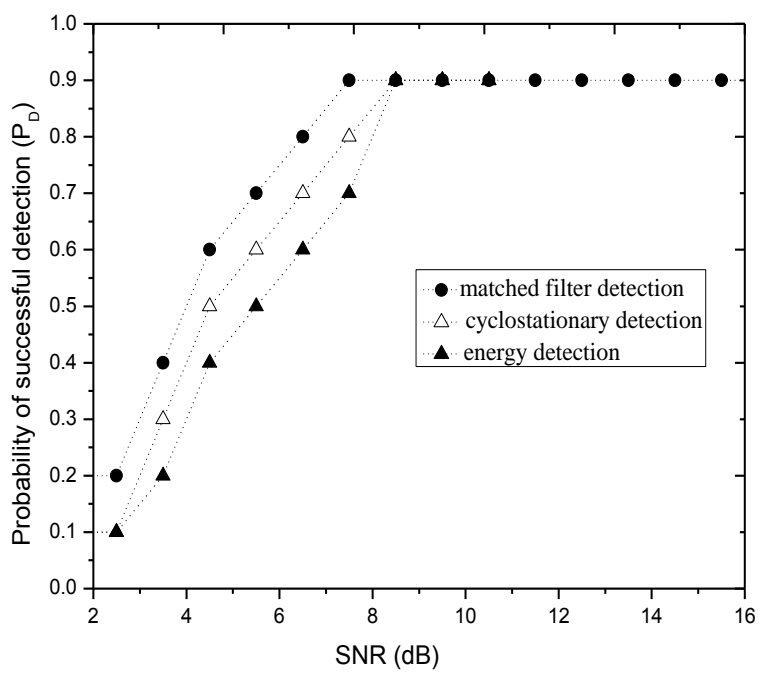

Figure 3: Probability of successful detection versus SNR

This figure represents the Probability of successful detection (PD) versus SNR for different spectrum sensing method. Here, the value of PD increases for different SNR values and for matched filter detection the PD value is much more compared to other two sensing technique.

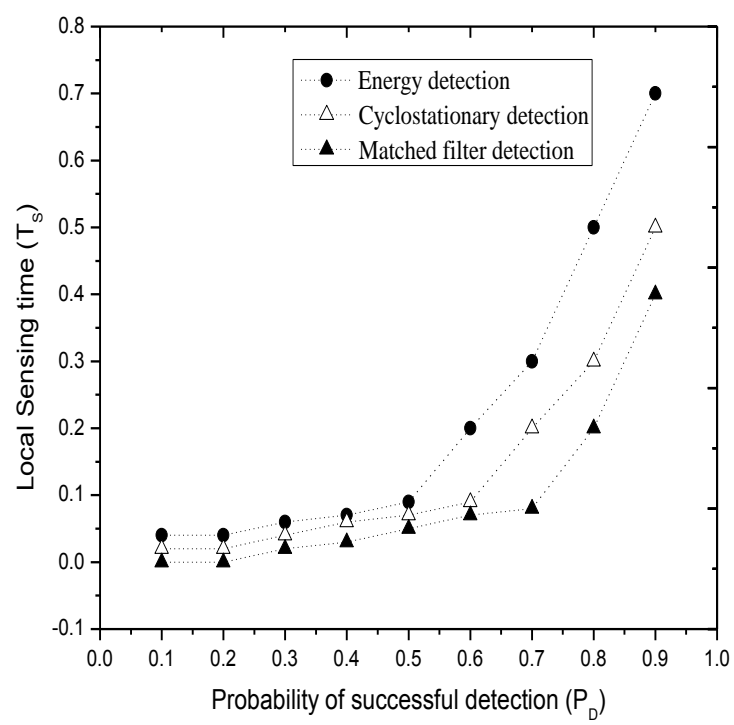

Figure4: Local Sensing time versus Probability of successful detection

This figure represents the local sensing time versus Probability of successful detection (PD) for different Spectrum Sensing methods. As they can see, the (TS) increases for different values of (PD). TS have maximum value for $\mathrm{ED}$ and minimum value for MD.

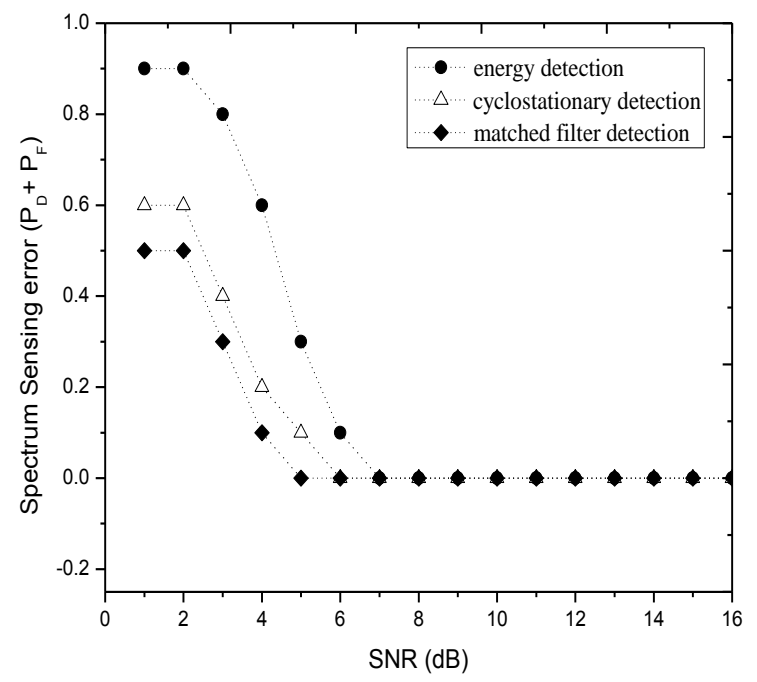

Figure 5: Spectrum sensing error versus SNR

The figure represents the spectrum sensing error versus SNR. As they can see the spectrum sensing error decreases as the SNR value increases. Because if SNR increases, signal power becomes more than noise power (Ps>PN) and making it easy to differ between presence and absence of PU. So sensing error decreases.

\section{CONCLUSION}

In this paper, they have performed comparative studies on different types of Spectrum Sensing method to conclude the best possible spectrum sensing method at low SNR value. The simulation result shows that matched filter is the best possible spectrum sensing method at low SNR as it gives a better performance for probability of successful detection and also takes less sensing time than the other two methods. It shows that probability of successful detection increases with the 
decrement of Probability of false alarm. Since its quality of sensing is far better therefore it causes less spectrum sensing error. The local sensing time for matched filter is also far below than the other sensing method. Hence it is the most efficient spectrum spectrum sensing technique.

\section{REFERENCES}

[1] Yoon, H. G., Chung, W. G., Jo, H. S., Lim, J., Yook, J. G., \& Park, H. K. (2006). Spectrum requirements for the future development of IMT-2000 and systems beyond IMT-2000. Journal of Communications and Networks, $8(2), 169-174$.

[2] Takagi, H., \& Walke, B. H. (Eds.). (2008). Spectrum requirement planning in wireless communications: Model and methodology for IMT-Advanced (Vol. 2). John Wiley \& Sons.

[3] Mitola, J. (2000). Cognitive radio---an integrated agent architecture for software defined radio.

[4] Pereira, J. N., Silva, P., Lima, P. U., \& Martinoli, A. (2013, November). An experimental study in wireless connectivity maintenance using up to 40 robots coordinated by an institutional robotics approach. In Intelligent Robots and Systems (IROS), 2013 IEEE/RSJ International Conference on (pp. 5073-5079). IEEE.

[5] Mitola, J., \& Maguire, G. Q. (1999). Cognitive radio: making software radios more personal. IEEE personal communications, 6(4), 13-18.

[6] Doyle, L. (2009). Essentials of cognitive radio. Cambridge University Press.

[7] Fette, B. A. (Ed.). (2009). Cognitive radio technology. Academic Press.

[8] Zhang, Y., Xu, G., \& Geng, X. (2008, September). Security threats in cognitive radio networks. In High Performance Computing and Communications, 2008. HPCC'08. 10th IEEE International Conference on (pp. 1036-1041). IEEE.

[9] Mitola, J., \& Maguire, G. Q. (1999). Cognitive radio: making software radios more personal. IEEE personal communications, 6(4), 13-18.
[10] Mitola, J. (2000). Cognitive radio---an integrated agent architecture for software defined radio.

[11] Tadaion, A. A. (2004). Notice of proposed rule making: unlicensed operation in the TV broadcast bands. ET Docket, (04-186).

[12] Marcus, M. J. (2005). Unlicensed cognitive sharing of TV spectrum: The controversy at the federal communications commission. IEEE Communications Magazine, 43(5), 24-25.

[13] Cordeiro, C., \& Challapali, K. (2005, November). Spectrum agile radios: utilization and sensing architectures. In New Frontiers in Dynamic Spectrum Access Networks, 2005. DySPAN 2005. 2005 First IEEE International Symposium on (pp. 160-169). IEEE.

[14] Yuan, Y., Bahl, P., Chandra, R., Chou, P. A., Ferrell, J. I., Moscibroda, T., ... \& Wu, Y. (2007, April). KNOWS: Cognitive radio networks over white spaces. In New Frontiers in Dynamic Spectrum Access Networks, 2007. DySPAN 2007. 2nd IEEE International Symposium on (pp. 416-427). IEEE.

[15] Ghasemi, A., \& Sousa, E. S. (2007, January). Optimization of spectrum sensing for opportunistic spectrum access in cognitive radio networks. In Consumer Communications and Networking Conference, 2007. CCNC 2007. 4th IEEE (pp. 1022-1026). IEEE.

[16] Datla, D., Rajbanshi, R., Wyglinski, A. M., \& Minden, G. J. (2007, April). Parametric adaptive spectrum sensing framework for dynamic spectrum access networks. In New Frontiers in Dynamic Spectrum Access Networks, 2007. DySPAN 2007. 2nd IEEE International Symposium on (pp. 482-485). IEEE.

[17] Zhao, Y., Morales, L., Gaeddert, J., Bae, K. K., Um, J. S., \& Reed, J. H. (2007, April). Applying radio environment maps to cognitive wireless regional area networks. In New Frontiers in Dynamic Spectrum Access Networks, 2007. DySPAN 2007. 2nd IEEE International Symposium on (pp. 115-118). IEEE. 